\title{
Longitudinal Study of Quality of Life in People with Advanced Alzheimer's Disease
}

\author{
Steven M. Albert, Ph.D., M.Sc., Diane M. Jacobs, Ph.D. \\ Mary Sano, Pb.D., Karen Marder, M.D. \\ Karen Bell, M.D., Davangere Devanand, M.D. \\ Jason Brandt, Ph.D., Marilyn Albert, Ph.D. \\ Yaakov Stern, Ph.D.
}

\begin{abstract}
The authors examined three indicators of health-related quality of life in people with advanced Alzheimer's disease ([AD]; $\mathrm{N}=150$ ): confinement to bome, null activity, and null positive affect, as reported by patient proxies. Dementia severity predicted timeto-onset for all three disease milestones in models that controlled for sociodemographic indicators, nursing home status, and death in the follow-up period. Patients whose dementia worsened over follow-up were more likely to reach each milestone. These outcomes represent key milestones in the care of patients; they are sensitive to disease progression, and they are likely to be useful for studying treatment in advanced $A D$. (Am J Geriatr Psychiatry 2001; 9:160-168)
\end{abstract}

\begin{abstract}
A key component in the investigation of health-related quality of life (HRQL) is "direct inquiry concerning aspects of their lives that most patients consider important." ${ }^{1}$ One central problem for HRQL assessment in people with advanced Alzheimer's disease $(\mathrm{AD})$ is the difficulty of such "direct inquiry." Severely affected patients (patients with Mini-Mental State Exam [MMSE] scores below $12^{2}$ or patients with more than moderate cognitive impairment ${ }^{3}$ ) cannot reliably complete selfreport questionnaires. It is unclear how patients with this level of dementia perceive subjective states. ${ }^{4}$ But we can agree with Lawton et al. that people with $\mathrm{AD}$ have good and bad moments, as well as likes and dislikes, that are evident to caregivers and provide a basis for investigating HRQL in $\mathrm{AD} .^{5}$ When patients cannot communicate $\mathrm{HRQL}$, investigators have to rely on proxy
\end{abstract}

reports or a patient's behavioral expression of subjective states.

A second problem is identifying domains or aspects of daily life likely to be important to patients in the presence of severely compromised cognition and functioning. The domains included in current measures vary considerably. Among other domains, Rabins et al. ${ }^{6}$ include "awareness of self" and "response to surroundings," and Brod et al., "aesthetic sense" and "feelings of belonging." Logsdon et al.s QOL-AD measure ${ }^{2}$ includes items assessing "energy level" and "ability to do things for fun." An alternative is to restrict HRQL to domains more closely linked to aspects of disease progression. This is the approach we have taken here, in which HRQL is defined in terms of restriction in activity and narrowing of affective expression.

Received March 22, 2000; revised June 2, 2000; accepted July 12, 2000. From the Gertrude H. Sergievsky Center and Department of Neurology, Columbia University, New York, NY. Address correspondence to Dr. Albert, Gertrude H. Sergievsky Center, P\&S Box 16, PH-19, Columbia University, 630 West 168th St., New York, NY 10032. e-mail: sma10@columbia.edu

Copyright (C) 2001 American Association for Geriatric Psychiatry 
In this study, we provide data on change in HRQL in a relatively large, longitudinal study of AD patients. We followed a well-characterized cohort of patients, mainly with advanced disease (moderate or more severe dementia), for up to 5 years, with HRQL assessment every 6 months. The goal was to determine the relationship between dementia severity and the risk of reaching basic HRQL milestones relevant to families of people with advanced $\mathrm{AD}$. The outcomes emerged from previous studies of HRQL in AD patients. ${ }^{5,8}$ and reflect our concern with identifying HRQL milestones that may be useful for clinical trials.

A further intent of our study was to identify HRQL milestones that would be useful for all advanced $\mathrm{AD}$ patients, that is, for patients remaining in the community and also for those who enter nursing homes. Knopman and colleagues ${ }^{9}$ have shown that nursing home placement is strongly related to increasing dementia severity and is thus an important HRQL milestone for severe dementia. We sought additional outcomes that might be useful even for patients who have entered nursing homes.

\section{METHODS}

\section{Measures}

HRQL Outcomes. In previous research, we developed a measure of HRQL for patients with AD based on proxy reports of patient activity and observed affect. ${ }^{5}$ We have designated the activity component of the instrument the "Patient Activity Scale-AD." It was originally derived from Teri and Logsdon's Pleasant Events Schedule$\mathrm{AD} .{ }^{10}$ We have designated the affect component of the instrument the "modified Apparent Emotion Scale," since it was derived from Lawton et al.'s Apparent Emotion scale. ${ }^{11}$ We have shown that the two measures are reliable in test-retest assessments and demonstrate adequate internal consistency, ${ }^{5}$ that family and institutional caregivers rate HRQL similarly for patients with similar grades of dementia, ${ }^{5}$ and that the measure is appropriate both in clinical and community-based samples. ${ }^{8}$

The Patient Activity Scale-AD elicits the frequency of 15 activities judged to be within the capacity of individuals with dementia receiving supervision and aid in daily activities. The measure includes five activities conducted outside the home (going outside; going to movies or other forms of entertainment; going to church, synagogue, or religious events; going shopping; going for a ride in a car) and 10 activities conducted mainly in the home (contact with a pet, getting together with family, talking to family or friends on the telephone, reading or being read to, listening to radio or watching television, exercising, playing games or puzzles, doing handicrafts, tending to plants or a garden, completing an additional unspecified task judged difficult by the caregiver). Proxies are asked if patients had the opportunity to participate in the activity in the previous 2 weeks, and, if so, how frequently they performed it. A summed activity score is calculated (number of activities performed of those the patient had the opportunity to perform).

For longitudinal analyses, we identified two activity outcomes. Patients were considered to have met the confined-to-home endpoint if they did not participate in any of the five activities that take place outside the home. Patients were said to have met the null-activity endpoint if they performed none or only 1 of the 15 activities. We included one activity in the null-activity endpoint because virtually all patients reported to participate in only one activity were "listening to the radio or watching television," the most passive of all the activities.

For the affect measure, proxies were asked to report how often patients expressed six affects over the previous 2 weeks. Each affect is specified by a clear set of facial and bodily indicators. The affects include both negative (anger, anxiety, depression) and positive (pleasure, interest, contentment) emotions. Proxies specify whether each affect occurred "never/can't tell," "only once," "2-6 times per week," " 1 or 2 times each day," or " 3 or more times each day." Because we have found that proxies have trouble identifying depression or contentment in patients with severe dementia, we defined outcomes on the basis of the other four affects. For longitudinal analyses, we identified one affect endpoint. We considered a patient to have null positive affect if proxies reported "never/can't tell" or only one occurrence of pleasure or interest over the previous 2 weeks. Null positive affect, then, should be interpreted as no or minimal expression of pleasure or interest.

Each of the outcomes was inspected over the course of follow-up to ensure that patients who met the endpoint at one follow-up continued to do so at subsequent assessments. All three outcomes demonstrated 
such stability. We rejected a "null negative affect" endpoint because of lack of such stability.

We did not combine the two indicators to produce a single score. The domains are conceptually distinct, and no compelling data are available for assigning weights to each domain to produce a composite. By identifying both an indicator of subjective states (affect) and behavior (activity), this approach follows an established model of quality-of-life investigation. ${ }^{12,13}$

Predictors of HRQL. At the same time that proxies reported patient activity and affect, severity of dementia in patients was assessed according to the Clinical Dementia Rating Scale (CDRS) ${ }^{14}$ and modified Mini-Mental State Exam (mMMS). ${ }^{15}$ The CDRS categorizes patients as having questionable, mild, moderate, severe, profound, or terminal dementia. Dementia severity ratings are based on assessment of patient status in six categories (memory, orientation, judgment and problemsolving, independence in community affairs, ability to function at home, and personal care competency). These ratings were derived from discussions with caregivers and, when possible, physician assessment of patients. The mMMS is based on the Folstein Mini-Mental State Exam (MMSE), and includes the Wechsler Adult Intelligence Scale (WAIS) Digit Span subtest, as well as additional attention/calculation, general knowledge, language, and construction items. Test scores range from 0 to 57 . The mMMS has been shown to be valid and reliable when used to assess $\mathrm{AD}$ patients. ${ }^{15}$

Because it is a measure of cognitive status alone, the mMMS offers an important advantage over the CDRS for examining HRQL outcomes that involve functional ability. Two of the HRQL outcomes (confinement to home and null activity) may partly overlap with categories of the CDRS (independence in community affairs and ability to function at home). For this reason, we used the mMMS score to examine the association between dementia severity and onset of the proposed HRQL outcomes. We reserved the CDRS only for an initial characterization of the cohort.

For longitudinal analyses, we categorized subjects into three mMMS groups: test scores of 0 (test floor), 1-29 (moderate-severe dementia), or $>30$ (mild dementia). We used this categorization of mMMS scores because this cohort study initially recruited subjects with mild dementia, in which mild dementia was defined as an mMMS score of 30 or above. A score of 30 on the mMMS is equivalent to a score of 16 on the Folstein MMSE.

Sociodemographic indicators examined in this research included age, sex, years of education, and residential status. We defined a group of patients living at home and a group in supervised living situations (nursing home, group home, non-nursing home medical facilities). We also determined, using the Columbia University Scale for Psychopathology in AD (CUSPAD), ${ }^{16}$ whether patients had psychiatric symptoms. Patients reported to have any symptom in the category of delusions, hallucinations, illusions, or agitated behavior were considered to have psychiatric symptoms. Finally, an important covariate in investigation of HRQL in advanced dementia is mortality over the follow-up period. Death is a possible indicator of progressive physical illness; and by including mortality in survival models, we are able to consider, at least to some degree, physical sources of poor HRQL. Physician examinations were unavailable for most patients.

\section{Procedures}

Assessment of HRQL began during the sixth to eighth follow-up evaluation of patients enrolled in the "Predictors Cohort," a multi-site longitudinal study of people with AD. Beginning in 1988, patients were recruited into the study from Columbia University, Johns Hopkins University, and Massachusetts General Hospital. Patients were drawn from memory disorders clinics, neurology practices, and clinical trials. Once enrolled, patients received detailed evaluations every 6 months. These evaluations were conducted by trained research assistants, who made visits to patients and their families, and by physicians, who conducted complete examinations at baseline and when a patient's medical status appeared to have changed. To be included in the cohort, patients had to meet DSM-III criteria for dementia along with NINDS-ADRDA criteria for probable AD.

Although the original cohort consisted of $236 \mathrm{pa}$ tients, only 150 subjects were active in the cohort when data collection for HRQL began. These 150 subjects have each had at least one HRQL evaluation, and nearly $90 \%$ have had two or more assessments. Median followup for the cohort for HRQL is 2.0 years, or five assessments; 44 patients (29.3\%) have had HRQL assessments every 6 months for 4 or more years, that is, nine or more assessments. In the follow-up period, 73 of the 150 sub- 
jects (48.7\%) have died; mMMS evaluations were available for 124 of the 150 patients.

To obtain information on patient activity and affect, a research assistant contacted patient proxies at each assessment interval. Most contacts were by telephone. For patients living at home, the family member designated as the patient contact-person from study inception served as proxy; in most cases, these informants were spouses or adult children. For patients residing in nursing homes, proxies could be either nursing home staff or family members. Preferentially, family contacts were sought, but in cases where family members did not feel they had enough contact to rate patients, we relied on nursing home staff.

Investigators obtained informed consent at the start of the study and at each follow-up, both from patients and family members. The protocol was approved by the Institutional Review Boards of Columbia University, Johns Hopkins University, and Massachusetts General Hospital.

\section{Statistical Methods}

We initially examined the cohort in terms of CDRS status. Subjects with mild, moderate, severe, and profound-terminal dementia were compared according to sociodemographic features, psychiatric symptoms, follow-up status, and subsequent mortality. These analyses involved one-way analysis of variance for continuous measures and chi-square tests for differences between proportions. The proportion of subjects in each dementia severity (CDRS) category who met the pre-specified HRQL outcomes was also examined, as this association can be considered a measure of the construct validity of the outcomes. Spearman rank-correlations were used to assess the relationship between HRQL indicators and psychiatric status.

We next identified subjects with follow-up data who were at risk for reaching the different HRQL outcomes. These subjects were included in longitudinal analyses. For these analyses, we used survival models and calculated the time to onset of HRQL milestones for the three mMMS groups. We used Kaplan-Meier models and the log-rank test to compare the risk of reaching the endpoint and Cox proportional-hazards models to establish the relative risk of reaching the endpoint, controlling for other predictors of poor HRQL. These predictors included age, sex, education, nursing home residence, and death in the follow-up period. Because information on medical status at each visit was not available for all patients, we included death as a predictor variable in these models; death in the follow-up period is a reasonable indicator of progressive physical illness and allows at least partial control for physical sources of poor HRQL. We also examined change in mMMS in these models, identifying a group whose dementia status worsened over follow-up and a group whose dementia did not worsen. This time-dependent covariate was also entered into models.

\section{RESULTS}

The modal CDRS category in this cohort at the first HRQL assessment was "moderate dementia;" 70 of the 150 subjects (46.7\%) fell into this category; $35 \%$ of the cohort, or 54 subjects, had more advanced dementia, although only one subject met criteria for terminal dementia. Features of the cohort at baseline, by CDRS status, are shown in Table 1.

At their initial HRQL assessment, subjects in the CDRS groups did not significantly differ in age or years of education. As expected, living situation was strongly related to dementia severity: $7.7 \%$ of the mild-dementia patients were residing in nursing homes or other forms of supervised living; the proportion increased to $40 \%$ in moderate dementia, $53.1 \%$ in severe dementia, and $59.1 \%$ in profound or terminal dementia subjects $(P<0.01)$. The proportion of subjects with psychiatric symptoms was lowest in the profound-terminal dementia group (18.2\%) and highest in the intermediate dementia severity groups $(46.9 \%-58.0 \% ; P<0.01)$.

Subjects with less severe dementia at their first HRQL evaluation had significantly longer follow-up $(P<0.001)$. This difference is entirely due to the greater risk of death among subjects with more severe dementia. Mortality ranged from $19.2 \%$ in mild dementia to $71.4 \%$ in the profound-terminal group $(P<0.05)$. Other sources of attrition did not differ by dementia status.

The quality-of-life indicators were significantly related to CDRS dementia severity, suggesting construct validity for the HRQL milestones. Patient proxies reported a mean of 5.0 activities in the mild, 3.3 in the moderate, 2.3 in the severe, and 1.0 in the profoundterminal dementia groups $(P<0.001)$. The proportion with null activity climbed from $8.0 \%$ in mild dementia to $22.1 \%$ in the moderate and $33.3 \%$ in the severe 
groups, and finally to $81.0 \%$ in the profound-terminal group $(P<0.001)$. Home confinement showed a similar trend $(P<0.001)$. Finally, no subject in the mild- and moderate-dementia groups met criteria for null positive affect, whereas $3.1 \%$ of the severe and $22.7 \%$ of the profound-terminal groups met such criteria $(P<0.001)$.

Psychiatric symptoms were inversely related to reported patient activity. Patients with psychiatric symptoms were significantly less likely to meet criteria for null activity (Spearman $r=-0.30 ; P<0.01$ ) and home confinement $(r=-0.35 ; P<0.01)$. By contrast, psychiatric symptoms were not significantly correlated with the positive-affect indicator $(r=-0.05$; NS). Null activ- ity and null positive affect were directly correlated $(r=0.31 ; P<0.01)$.

\section{Longitudinal Findings}

By the end of the follow-up period (or, for those who died, their last assessment), 6.1\% of the cohort had mild and 22.4\%, moderate dementia by CDRS; most of the cohort had either severe $(32.0 \%)$ or profound (33.3\%) dementia. An additional 6.1\% were classified as having terminal dementia.

Table 2 summarizes HRQL longitudinal findings for the cohort according to dementia severity by mMMS scores. As stated earlier, 124 patients were available for

TABLE 1. AD subjects with QOL assessment, by dementia severity: baseline indicators and follow-up status

\begin{tabular}{|c|c|c|c|c|}
\hline & Mild $(n=26)$ & Moderate $(n=70)$ & Severe $(n=32)$ & Profound-Terminal $(n=22)$ \\
\hline \multicolumn{5}{|l|}{ Sociodemographics } \\
\hline Female, \% & 34.6 & 67.1 & 65.6 & $59.3^{*}$ \\
\hline Age, years $\pm S D$ & $74.4 \pm 6.7$ & $76.2 \pm 7.9$ & $74.3 \pm 9.1$ & $71.8 \pm 8.5$ \\
\hline Education, years $\pm \mathrm{SD}$ & 14.4 & 12.5 & 13.2 & 13.2 \\
\hline Nursing home resident, \% & 7.7 & 40.0 & 53.1 & $59.1^{* *}$ \\
\hline Psychiatric symptoms, \% & 38.5 & 58.0 & 46.9 & $18.2^{* * *}$ \\
\hline \multicolumn{5}{|l|}{ Follow-up status } \\
\hline Months, mean $\pm \mathrm{SD}$ & $38.5 \pm 14.7$ & $28.9 \pm 18.6$ & $25.9 \pm 20.0$ & $16.6 \pm 18.7^{* * * *}$ \\
\hline 6-month follow-up, \% & 100.0 & 94.3 & 87.5 & $72.7^{* * *}$ \\
\hline Mortality over follow-up, \% & 19.2 & 48.6 & 59.4 & $71.4^{*}$ \\
\hline \multicolumn{5}{|l|}{ QOL indicators } \\
\hline Activities, mean \pm SD & $5.0 \pm 2.8$ & $3.3 \pm 2.0$ & $2.3 \pm 1.7$ & $1.0 \pm 1.3^{* * * *}$ \\
\hline Null activity, \% & 8.0 & 22.1 & 33.3 & $81.0^{* * * *}$ \\
\hline Home confinement, \% & 4.1 & 38.4 & 31.5 & $86.4^{* * * *}$ \\
\hline Null positive affect, \% & 0.0 & 0.0 & 3.1 & $22.7^{* * * *}$ \\
\hline \multicolumn{5}{|c|}{$\begin{array}{l}\text { Note: } \mathrm{AD}=\text { Alzheimer's disease; } \mathrm{QOL}=\text { quality of life; } \mathrm{SD}=\text { standard deviation. } \\
\text { Dementia severity was defined by the Clinical Dementia Rating Scale (CDRS). }{ }^{14} \text { Psychiatric symptoms: presence of delusions, hallucinations, } \\
\text { illusions, or agitation. Activities: number of simple, caregiver-cued activities (range: } 0-15) \text { performed at least once over previous } 2 \text { weeks; five } \\
\text { of the activities are performed outside the home. Null activity: performance of none or one such activity only. Home confinement: no activities } \\
\text { performed outside the home. Null positive affect: no or minimal expression of pleasure or interest over the previous } 2 \text { weeks. } \\
\quad{ }^{*} P<0.05 ;{ }^{* *} P<0.01 ;{ }^{* * *} P<0.001 \text { by one-way analysis of variance (means) or } \chi^{2} \text { (proportions). }\end{array}$} \\
\hline
\end{tabular}

TABLE 2. Quality-of-life outcomes and initial dementia severity

\begin{tabular}{|c|c|c|c|c|}
\hline & Home Confinement & Null Activity & Null Positive Affect & $\begin{array}{l}\text { Median Length of } \\
\text { Follow-Up: Months }\end{array}$ \\
\hline \multicolumn{5}{|c|}{ Prevalent cases, $n$} \\
\hline mMMS $\geq 30$ & 12 & 6 & 0 & \\
\hline mMMS $<29$ & 28 & 17 & 0 & \\
\hline $\mathrm{mMMS}=0$ & 13 & 8 & 2 & \\
\hline \multicolumn{5}{|c|}{ Subjects at risk, $n$ (\% reaching outcome) } \\
\hline mMMS $\geq 30$ & $15 / 28(53.6)$ & 3/34 (8.8) & $1 / 40(2.5)$ & 42 \\
\hline mMMS $<29$ & $24 / 37(64.9)$ & $21 / 48(43.8)$ & $7 / 65(10.8)$ & 33 \\
\hline $\mathrm{mMMS}=0$ & $4 / 6(66.7)$ & $6 / 11(54.5)$ & $4 / 17(23.5)$ & 24 \\
\hline \multicolumn{5}{|c|}{$\begin{array}{l}\text { Note: } \text { mMMS = modified Mini-Mental State Exam. } \\
\text { Home confinement: over previous } 2 \text { weeks, subject did not go out in car, shop, go outside for walk, visit place of worship, or attend } \\
\text { entertainment outside the home. Null activity: over previous } 2 \text { weeks, subject performed none of } 15 \text { activities or only } 1 \text { activity (listening to } \\
\text { adio or watching television). Null positive affect: over previous } 2 \text { weeks, subject never expressed pleasure or interest, or expressed these } \\
\text { ffects only once. }\end{array}$} \\
\hline
\end{tabular}


these analyses. Patients who had already met criteria for the endpoint at their first HRQL assessment were considered prevalent cases and are shown in the top half of the table; these subjects were excluded from longitudinal analyses.

In keeping with the moderate-to-severe dementia status of the cohort, by the time of their first HRQL assessment, $42.7 \%(53 / 124)$ had already reached the homeconfinement endpoint; $25 \%$ (31/124), the null-activity endpoint; and $1.6 \%(2 / 124)$, the null positive affect endpoint.

Among subjects at risk for reaching the HRQL milestones, initial dementia status was associated with onset of poor HRQL. For example, 53.6\% of subjects with mMMS $\geq 30,64.9 \%$ of subjects with mMMS scores between 1 and 29 , and $66.7 \%$ of subjects scoring at test floor met the home-confinement outcome over the fol-

FIGURE 1. Risk of home confinement, by initial dementia severity

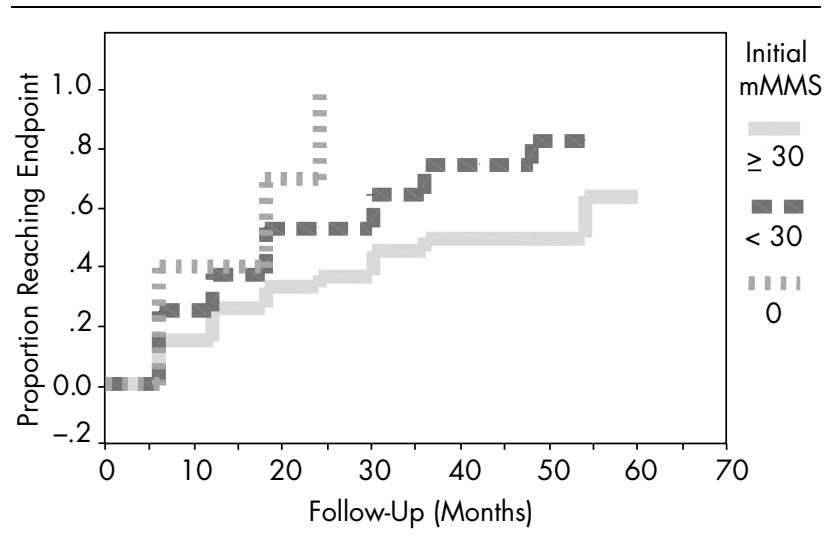

Note: $n=71 ; P<0.04$ by log-rank test.

FIGURE 2. Risk of onset of null activity, by initial dementia severity

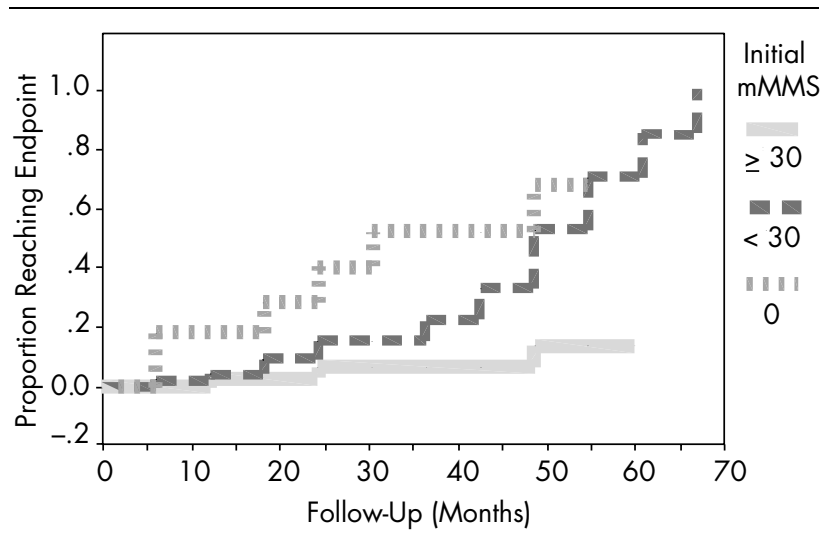

Note: $n=93 ; P<0.01$ by log-rank test. low-up period. For null activity, the proportions were $8.8 \%, 43.5 \%$, and $54.5 \%$, respectively; and for null positive affect, the proportions were $2.5 \%, 10.8 \%$, and $23.5 \%$, respectively.

Kaplan-Meier plots, which take into account timeto-onset, show that dementia severity (by mMMS) at the initial assessment was associated with significantly earlier onset for each outcome $(P<0.04$ for home confinement and $P<0.01$ for null activity and null positive affect milestones). These plots are shown in Figure 1, Figure 2, and Figure 3 .

These risk estimates are based on incident cases; that is, patients had been performing the activity or demonstrated positive affect and later showed impaired HRQL by these indicators. The analyses take mortality into account by censoring subjects who died over the follow-up period. Within 2 years of their initial assessment, all patients initially scoring at the floor of the mMMS were confined to home, compared with half the patients with scores less than 30 and $35 \%$ of patients with scores 30 or greater (Figure 1). At 2 years, $40 \%$ of patients initially scoring at the mMMS floor had met the null-activity endpoint, compared with only $10 \%$ of patients with scores less than 30 and $5 \%$ of patients with scores of 30 or greater (Figure 2). Onset of null positive affect was a later event, with differences in incidence among the dementia-severity groups evident only after 2 years of follow-up (Figure 3 ).

Cox proportional-hazards models for the three HRQL milestones showed that initial dementia severity was a significant predictor in models that adjusted for sociodemographic indicators (age, sex, education), initial residence status (nursing home or other assisted liv-

FIGURE 3. Risk of null positive affect, by initial dementia severity

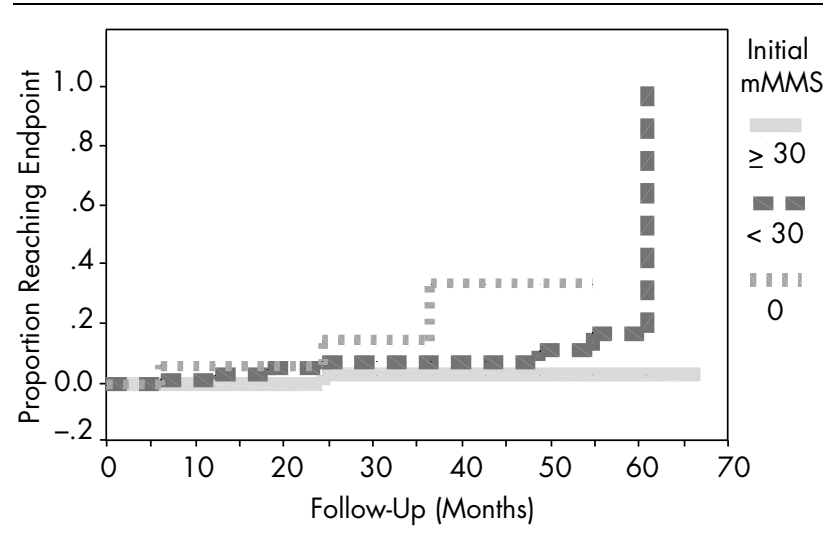

Note: $n=122 ; P<0.01$ by log-rank test. 
ing facility vs. community residence), and mortality over follow-up. We did not include initial psychiatric status in these models because psychiatric symptoms were quite variable across assessment intervals. Table 3 shows results for the proportional-hazards models.

Residence in a nursing home or assisted-living facility was a strong predictor of home confinement (relative risk $[R R]=2.2 ; 95 \%$ confidence interval $[\mathrm{CI}]: 1.0-$ 5.2); yet even with this predictor included in the model, patients scoring 30 or greater on the mMMS were only one-third as likely to reach this endpoint when compared with subjects scoring at the mMMS floor. Death over the follow-up period was a significant predictor for the onset of null activity $(\mathrm{RR}=3.0$; 95\% CI: 1.3-6.8). Yet even with mortality status included in the model, patients scoring 30 or above on the mMMS faced only one-tenth of the risk of reaching this outcome when compared with subjects scoring at test floor. A gradient in risk associated with dementia severity was evident for all three milestones.

Finally, we examined whether patients whose dementia worsened over follow-up were more likely to reach the HRQL milestones. We defined five groups according to change in mMMS scores: subjects at test floor at all assessments, subjects whose initial test score was 1-29 but later declined to test floor, subjects whose initial test score was 1-29 and remained in this range, subjects whose initial test score was $\geq 30$ but declined to 1-29 or test floor, and subjects whose test score was $\geq 30$ on all assessments. We then examined the proportion of subjects in each group who reached the HRQL milestones. Results are shown in Table 4.

Subjects whose dementia worsened over follow-up were more likely, for the most part, to reach the HRQL milestones. For example, in the group whose initial
mMMS score was 1-29, $91.7 \%$ of those whose score declined eventually were confined to home, compared with $52.0 \%$ of those whose score did not change to this degree. For subjects whose initial score was $\geq 30$, $70.6 \%$ of those whose scores declined reached this endpoint, compared with $27.3 \%$ of those with more stable scores. Kaplan-Meier plots, shown in Figure 4, indicate a clear gradient in risk of home confinement related to declining cognitive performance. Similar findings were evident for the other HRQL milestones, as shown in Table 4 .

\section{DISCUSSION}

This research is valuable for demonstrating the usefulness of three health-related quality-of-life (HRQL) milestones for assessing progression and perhaps treatment of advanced AD. Home confinement, null activity, and null positive affect are straightforward indicators of HRQL: they are easily rated by proxies, strongly related to dementia severity in cross-sectional and longitudinal analyses, relevant even in late stages of dementia (i.e., after nursing home admission), and inherently meaningful to patients and families. The last point is worth special consideration. Not being able to leave the home even with caregiver support and supervision is a clear indicator of how impairing cognitive deficit may be. The same is true for the null activity and null positive affect milestones. Our models controlled for other sources of impairment that might increase the risk of these outcomes, such as death over follow-up (an indicator of physical illness), sociodemographic indicators, and environmental constraints on activity and engage-

TABLE 3. Relative risk ( $95 \%$ confidence interval) of reaching HRQL outcomes

\begin{tabular}{|c|c|c|c|}
\hline & Onset of Home Confinement & Onset of Null Activity & Onset of Null Positive Affect \\
\hline \multicolumn{4}{|l|}{ mMMS } \\
\hline Floor & 1.0 (ref) & 1.0 (ref) & 1.0 (ref) \\
\hline$<30$ & $0.4(0.1-1.5)$ & $0.6(0.2-1.6)$ & $0.3(0.1-1.1)$ \\
\hline$\geq 30$ & $0.3(0.8-1.1)$ & $0.1(0.02-0.5)$ & $0.04(0.01-0.5)$ \\
\hline \multicolumn{4}{|l|}{ Residence } \\
\hline Community & 1.0 (ref) & 1.0 (ref) & 1.0 (ref) \\
\hline Assisted living & $2.2(1.0-5.2)$ & $0.8(0.4-2.0)$ & $0.7(0.2-2.9)$ \\
\hline \multicolumn{4}{|l|}{ Mortality } \\
\hline Alive & 1.0 (ref) & 1.0 (ref) & 1.0 (ref) \\
\hline Died & $1.5(0.8-2.9)$ & $3.0(1.3-6.8)$ & $2.2(0.6-8.9)$ \\
\hline$n$ & 71 & 93 & 122 \\
\hline
\end{tabular}


ment, such as nursing home placement. Thus, we conclude that the milestones are likely to be sensitive indicators of disease progression and treatment response.

The outcomes identified in this research help satisfy the need to identify domains or aspects of life likely to be important to patients with severely compromised cognition and functioning, as well as to their families. Undoubtedly, there are other aspects of HRQL that are relevant to this population, and measurement efforts should be directed toward these, as well; but the proposed measures have the virtue of simplicity, relevance to patients and caregivers, and strong association with disease progression across the spectrum of dementia severity.

Onset of the HRQL milestones analyzed here helps show the impact of $\mathrm{AD}$ on patients and their families as patients progress from mild or moderate to more severe stages of dementia. For example, we have shown that within 2 years of follow-up, $40 \%$ of patients who began follow-up with scores at the floor of the mMMS met the null-activity milestone, compared with $10 \%$ of patients with scores less than 30 and $5 \%$ of patients with scores of 30 or greater. These findings give families an idea of the time-course of the disease and when they can ex-

FIGURE 4. Risk of home confinement, by change in dementia severity

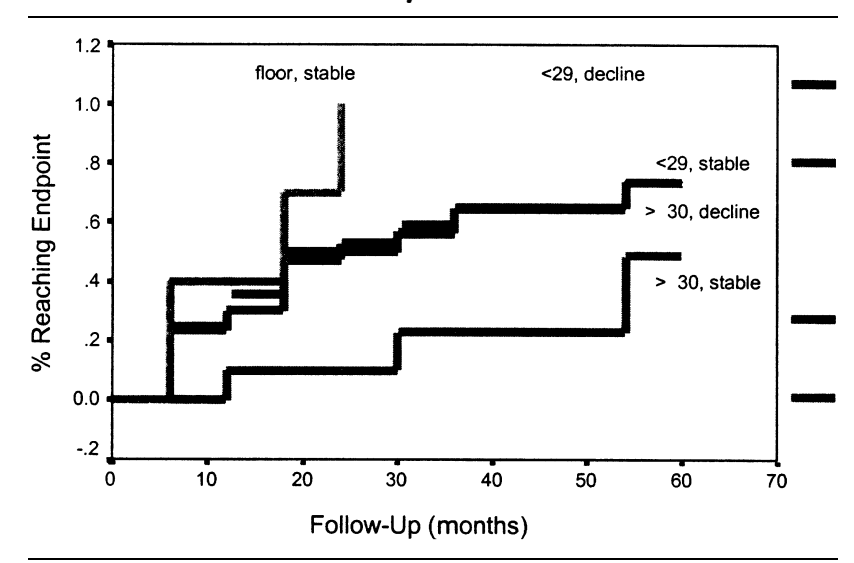

pect patients to reach milestones that require new caregiving strategies. Similarly, we found that patients whose mMMS scores declined over follow-up were at far greater risk of reaching the HRQL outcomes than patients with stable scores. However, caution must be exercised in using these findings to make predictions for any particular patient. As these data show, there is considerable variability across patients.

Notably, in survival models, the relationship between mMMS and onset of home confinement was only marginally significant; this stands in contrast to the strong association between mMMS and both the nullactivity and null positive affect outcomes (as shown in Table 3). This difference suggests that home confinement reflects factors other than cognitive decline, such as patient mobility status, caregiver motivation to arrange outings, features of dwellings and neighborhoods, and access to transportation.

The association between dementia severity and the risk of reaching such HRQL outcomes may help explain the extremely low ratings given to states of dementia by caregivers and lay raters. In studies of "health utilities," that is, the extent to which one health state is preferred over another, advanced $\mathrm{AD}$ is often given ratings similar to or worse than death (i.e., close to 0 , as opposed to 1.0, which indicates normal or optimal health). Caregivers completing the Health Utilities Index with regard to $\mathrm{AD}$ patients gave scores of 0.69 for mild $\mathrm{AD}, 0.53$ for moderate, 0.38 for severe, 0.27 for profound, and 0.14 for terminal dementia. ${ }^{17} \mathrm{~A}$ second study, using a time trade-off method, confirmed these values; in this study, clinicians gave states of mild dementia a utility of 0.67 and severe dementia a utility of $0.31 .^{18}$ Finally, caregivers in a third study, using the Quality of Well-Being scale, reported utilities of $0.40-$ 0.50 for $\mathrm{AD}$ patients who were "unable to do simple tasks." ${ }^{3}$ All three studies confirm the severe HRQL impact of advanced $\mathrm{AD}$.

TABLE 4. Proportion reaching quality-of-life outcomes, by change in mMMS scores over follow-up

\begin{tabular}{lccc}
\hline mMMS Group & $\begin{array}{c}\text { Onset of Home Confinement } \\
(\boldsymbol{n}=\mathbf{7 1})\end{array}$ & $\begin{array}{c}\text { Onset of Null Activity } \\
(\boldsymbol{n}=93)\end{array}$ & $\begin{array}{c}\text { Onset of Null Positive Affect } \\
(\boldsymbol{n}=\mathbf{1 2 2})\end{array}$ \\
\hline Test floor, all assessments & 67.7 & 54.5 & 31.6 \\
1-29; decline & 91.7 & 40.0 & 10.0 \\
1-29; stable & 52.0 & 46.9 & 11.1 \\
$\geq 30$; decline & 70.6 & 16.7 & 4.5 \\
$\geq 30 ;$ stable & 27.3 & 0.0 & 0.0 \\
\hline Note: $\mathrm{mMMS}$ = modified Mini-Mental State exam. & & \\
\hline
\end{tabular}


The outcomes identified in this research are valuable for allowing further specification of the HRQL impact of advanced dementia. For example, it would be valuable to know more about variation in the onset of the HRQL outcomes among patients with initially similar dementia severity. Differences in risk might reflect variation in disease course, different caregiving environments, different therapies, or some other factor.

A further consideration in this research is the relative independence of the HRQL outcomes from patient psychiatric status. In our cross-sectional analyses, positive affect and psychiatric symptoms were not related, suggesting that the two represent different components of psychological experience in patients with AD. The relative preservation of positive affect in the presence of psychopathology suggests that we need to examine positive aspects of mental health in these patients alongside such traditional indicators as agitation, depression, and delusions.

We conclude that health-related quality of life in advanced AD is likely to be valuable as an outcome for measuring disease progression and treatment outcomes. Because the outcomes are strongly associated with dementia severity, they may serve as important indicators of the efficacy of treatments for the disease and should be considered alongside such traditional outcomes as cognitive performance, psychopathology, and functional status.

This research was supported by grants from NIH (AGO7370 and RRO0645) and the Charles S. Robertson Gift for Alzheimer's Disease from the Banbury Fund.

\section{References}

1. Guyatt GH, Jaeschke RJ: Reassessing quality-of-life instruments in the evaluation of new drugs. Pharmacoeconomics 1997; 12:621626

2. Logsdon RG, Gibbons LE, McCurry SM, et al: Quality of life in Alzheimer's disease: patient and caregiver reports. Journal of Mental Health and Aging 1999; 5:21-32

3. Kerner DN, Patterson TL, Grant I, et al: Validity of the Quality of Well-Being scale for patients with Alzheimer's disease. Journal of Aging and Health 1998; 10:44-61

4. Lawton MP, Van Haitsma K, Perkinson M, et al: Observed affect and quality of life in dementia: further affirmations and problems, in Assessing Quality of Life in Alzheimer's Disease. Edited by Albert SM, Logsdon RG. New York, Springer, 2000, pp 95-110

5. Albert SM, Castillo-Castenada C, Sano M, et al. Quality of life in patients with Alzheimer's disease as reported by patient proxies. J Am Geriatr Soc 1996; 44:1342-1347

6. Rabins PV, Kasper JD, Kleinman L, et al: Concepts and methods in the development of the ADRQL: an instrument for assessing health-related quality of life in persons with Alzheimer's disease. Journal of Mental Health and Aging 1999; 5:33-48

7. Brod M, Stewart AL, Sands L: Conceptualization of quality of life in dementia. Journal of Mental Health and Aging 1999; 5:7-20

8. Albert SM, Castillo-Casteneda C, Jacobs DM, et al: Proxy-reported quality of life in Alzheimer's patients: comparison of clinical and population-based samples. Journal of Mental Health and Aging 1999; 5:49-58

9. Knopman DS, Berg JD, Thomas R, et al: Nursing home placement is related to dementia progression: experience from a clinical trial. Neurology 1999; 52:714-718
10. Teri L, Logsdon RG: Identifying pleasant activities for Alzheimer's disease patients: the Pleasant Events Schedule-AD. Gerontologist 1991; 31:124-127

11. Lawton MP, van Haitsma K, Klapper J: Observed affect in nursing home residents with Alzheimer's disease. J Gerontol Psychol Sci 1996; 51B:P3-P14

12. Erickson P, Wilson R, Shannon I: Years of healthy life. Healthy People 2000: Statistical Notes, Number 7. Atlanta, GA: U.S. Department of Health and Human Services, Centers for Disease Control: National Center for Health Statistics (April 1995)

13. Albert SM: Assessing quality of life in chronic care populations, in Measurement in Elderly Chronic Care Populations. Edited by Teresi JA, Lawton MP, Holmes D, et al. New York, Springer, 1997, pp 210-227

14. Hughes CP, Berg L, Danziger WL: A new clinical scale for the staging of dementia. Br J Psychol 1982; 140:556-572

15. Stern Y, Sano M, Paulson J, et al: Modified Mini-Mental State Exam: validity and reliability (abstract). Neurology. 1987; 37(suppl 1): 179

16. Devanand DP, Miller L, Richards $M$, et al: The Columbia University Scale for Psychopathology in Alzheimer's Disease. Arch Neurol 1992; 15:369-377

17. Newman PJ, Kuntz KM, Leon J, et al: Health utilities in Alzheimer's disease: a cross-sectional study of patients and caregivers. Med Care 1999; 37:27-32

18. Sano M, Albert SM, Tractenberg R, et al: Developing utilities: quantifying quality of life for stages of Alzheimer's disease as measured by the Clinical Dementia Rating Scale. Journal of Mental Health and Aging 1999; 5:59-68 